\title{
Where now for plasmonics?
}

\author{
Developing useful methods to control light-matter interactions at the nanoscale requires an \\ appreciation of the needs of industry and innovative approaches that go beyond plasmonics.
}

According to a recent analysis, the field of plasmonics has now reached the slope of enlightenment, a phase of rational assessment of the real potential of the field that should lead to technologies of general, societal usefulness (U. Guler, A. V. Kildishev, A. Boltasseva and V. M. Shalaev, Faraday Discuss. 178, 71-86; 2015). Gone is the widespread initial excitement that emerged around the turn of this century and led to considerable hype surrounding the potential of the field. As with many scientific endeavours, it seems as though this phase of inflated expectations is a necessary rite of passage: a collective effort in which researchers explore the possibilities offered by a new vein of research dotted with amazing discoveries, such as, in the case of plasmonics, extraordinary optical transmission, new materials with negative refractive index, and spasers, to name a few. While some people may come to the realization that not all that glitters is gold (quite literally in this case) earlier than others, the significant resources invested in this exciting phase is the basis for building a solid conceptual foundation for the field and shaping a new scientific community. Inevitably, many of these insights can end up unveiling fundamental limitations and dead-ends, which, in plasmonics, are associated with optical losses and the inability to modulate the optical signal using noble metals. Nonetheless, the knowledge acquired is also valuable for developing new strategies to counteract them. In this issue of Nature Nanotechnology, we look at some of these strategies and, in particular, the alternative ways researchers are exploring to achieve control of the light-matter interaction at the nanoscale.

Probably the most disruptive strategy is to get rid of metals altogether and use all-dielectric materials. In a Review article on page 23, Saman Jahani and Zubin Jacob give an overview of the physics of dielectric resonators: nanostructures with a high refractive index that can focus an electromagnetic field at the nanoscale through directional Mie resonances, instead of plasmons. Recent investigations have shown that by using dielectric resonators it is possible to achieve a full range of optical responses covering all four quadrants

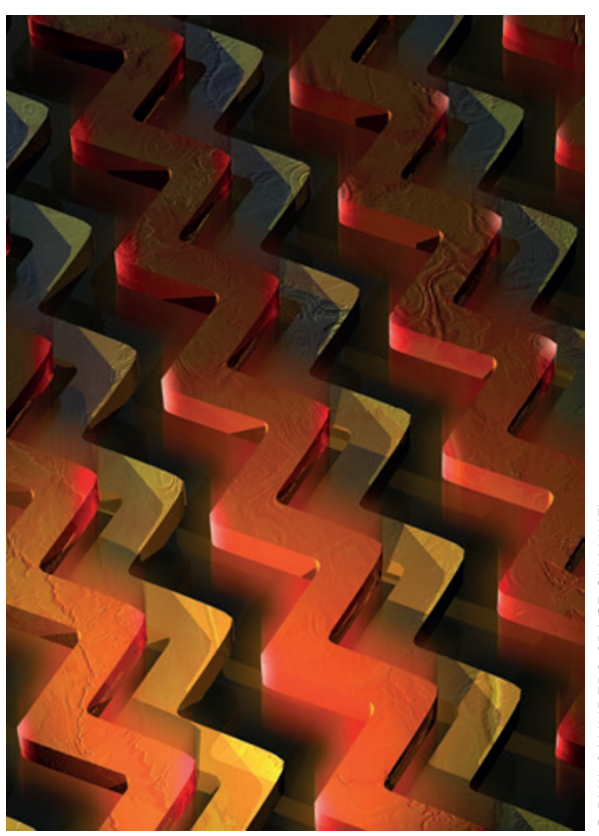

that can work at various wavelengths in the mid-IR to terahertz regime, and goes as far as envisioning the rational design of crystalline hybrids - new compound materials that would extend the applicability of the approach to a broader range of wavelengths.

An alternative idea to convert the current knowledge in the field of plasmonics into practical applications is to exploit the various forces at play at the nanoscale. In a Perspective on page 16, Nikolay Zheludev and Eric Plum discuss how electromagnetic interactions, such as Coulomb, Lorentz and Ampère forces, can be used to actuate metasurfaces to modulate optical signals. Large optical nonlinearities, electro-optical and magneto-optical effects have already been demonstrated and dynamic control over light with subwavelength resolution seems within reach. This technology, which is CMOS-compatible, can already deliver modulation frequencies in the megahertz range and could become relevant in optical telecommunication. As a demonstration of the generality of the physics at play, fast actuation can also be achieved using thermal, optical and magnetic interactions.

\section{Probably the most disruptive strategy is to get rid of metals altogether and use all-dielectric materials.}

These are, of course, only a few of the possibilities that have come to the fore in recent years as the field of plasmonics and metamaterials has matured, and our collection of articles is not intended to be comprehensive. However, one thing seems clear: recognizing the fundamental issue of optical losses has forced the community to come up with creative solutions, which have emerged by investigating unconventional approaches or looking out for materials, systems and interactions that may complement the strengths of plasmonics. In this phase of enlightenment, it is also imperative to maintain a strong appreciation for the technological needs of industry so that the field can ultimately fulfil its full potential. 